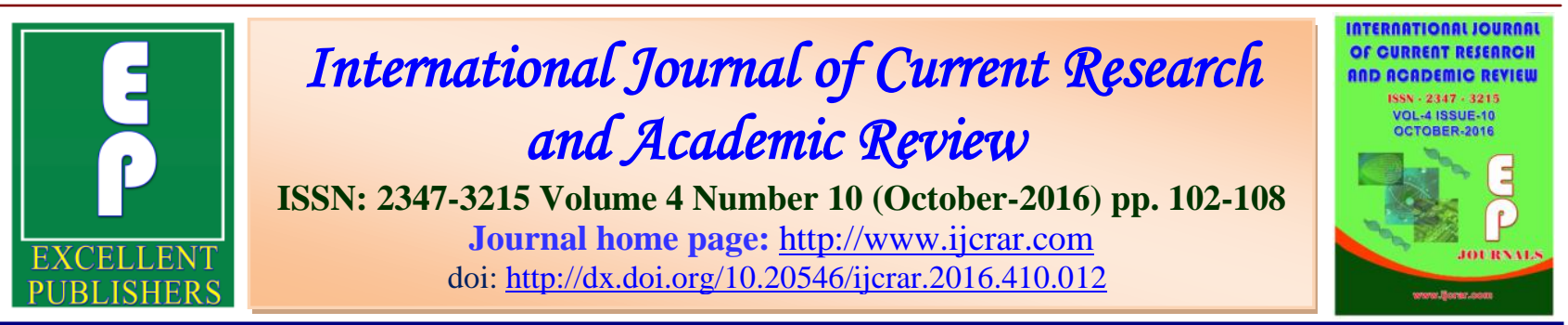

\title{
The Relationship between Preoperative Serum Levels of HE4 and Epithelial Ovarian Cancer
}

\author{
Fatemeh Cheraghi $^{1}$, Zohreh Moini ${ }^{2 *}$ and Mojgan Barati ${ }^{3}$ \\ ${ }^{1}$ Department of Obstetrics and Gynecology, Fertility Infertility and Perinatology Research \\ Center, Imam Khomeini Hospital, Ahvaz Jundishapur University of Medical Sciences, Ahvaz, \\ Iran \\ ${ }^{2}$ Student Research Committee, Ahvaz Jundishapur University of Medical Sciences, Ahvaz, \\ Iran \\ ${ }^{3}$ Fertility, Infertility and Perinatology Research Center, Imam Khomeini Hospital, School of \\ Medicine, Ahvaz Jundishapur University of Medical Sciences, Ahvaz, Iran \\ *Corresponding author
}

KEYWORDS
Cancer,
epithelial ovarian
cancer,
HE-4

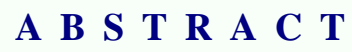

Ovarian cancer is the most common malignancy in the world and the fifth leading cause of death from cancer among women. HE-4 or (Human Epididymis protein 4) is a new marker introduced for diagnosis and follow-up of ovarian cancer. The aim of this study is to examine the association between preoperative serum levels of the marker of HE4 and epithelial ovarian cancer based on FIGO system to evaluate this marker in predicting the ovarian cancer stage. This analytical retrospective study is conducted on 62 patients with ovarian masses hospitalized in Ahwaz Imam Khomeini Hospital for surgery. The records of the patients who had been hospitalized within the years of 2011-2013 was analyzed and the HE4 serum level was measured by ELISA method and also the information in the patient records were recorded in demographic questionnaires. The data were analyzed with SPSS software version 18. In this study 62 patients with ovarian masses were studied among whom 26 cases $(41.9 \%)$ were postmenopausal. Statistical test results showed that the HE-4 levels in postmenopausal women had no significant difference with other patients ( $\mathrm{P}>0.05)$, but the mean HE-4 level in different stages showed that by increasing the stage the HE-4 level is increased significantly $(\mathrm{P}=0.007)$. This increase was also applied in patients' grade and HE-4 was significantly higher in severe cases the $(\mathrm{P}=0.003)$. Also HE-4 level was significantly higher in malignant patients than the benign patients $(\mathrm{P}=0.001)$. Based on the results obtained in this study it can be concluded that HE4 is a good diagnostic marker for differentiating benign and malignant ovarian tumors and more importantly in malignant patients its level is increased as the grade and stage of the disease increase and thus it is recommended to apply it in patients with a diagnosis of ovarian tumors. 


\section{Introduction}

Today cancer is a widespread public health problem that affects different tissues (Firoozabadi et al., 2015; Rahmani et al., 2016; Mohammad et al., 2015). Ovarian cancer is the most common malignancy in the world and the fifth leading cause of death caused by cancer among women. In Iran ovarian cancer is the eighth most common cancer in women and its five-year survival rate has been reported as $6 \%$ (Arab et al., 2010). Ovarian cancer has multiple histopathologic types. In a study in Iran the prevalence of these forms is reported as $81.6 \%$ epithelial, $12.2 \%$ germ cell and $5.1 \%$ granulosa cell. The average age of ovarian cancer in Iran is lower than the Western countries and a high percentage of patients are diagnosed at the advanced stages (Khodabakhshi et al., 2007). Ovarian cancer mortality rate is around $75 \%$ but its diagnosis in early stages of the disease reduces the mortality rate to about $10 \%$. Strategies to diagnose the disease in early stages require tests with high sensitivity and specificity over $75 \%$ and $99.6 \%$ so that the positive predictive value is reduced to a minimum level of $10 \%$. The early stages of ovarian cancer has ambiguous are non-specific symptoms; therefore most patients refer at the advanced stages of the disease and thus several tumor markers are introduced for screening and diagnosis of cancer particularly ovarian cancer (IIana, 2008).

HE-4 or (Human Epididymis protein 4) is a new marker introduced for diagnosis and follow-up of ovarian cancer (Moore et al., 2008). This marker is discovered by comparing RNA sequences expressions in normal and malignant ovarian epithelial cells. Studies have demonstrated that the concurrent use of CA125 and HE4 markers had the sensitivity equivalent to $76.46 \%$ and specificity equivalent to $100 \%$ to diagnose and investigate recurrent ovarian cancer.
Among the few studies in this area, in one study the high levels of HE-4 have been associated with the advanced stages of tumor and serous ovarian cancer (Paek et al., 2011). Moreover the use of this marker is developing for staging and invading the endometrial cancer myometrium (Moore et al., 2011). The importance of staging ovarian cancer is more specific than any other cancers because the microscopic metastases are serious problems in the preoperative treatment. Unfortunately, in Iran and many other countries, staging is no fully exercised due to technical issues and therefore the lack of proper staging leads to inappropriate treatment of many patients. The study on the tumor markers in addition to the early diagnosis, follow up after diagnosis and treatment could predict the tumor stage based on serum levels is increasing (Arab et al., 2010). In line with this objective, in this study the association between preoperative serum levels of the marker of HE4 and epithelial ovarian cancer based on FIGO system to evaluate this marker in predicting the ovarian cancer stage is studied to evaluate this marker in predicting the ovarian cancer stage and help the early diagnosis of the disease in early stages and treat the patient more effectively.

\section{Materials and Methods}

After obtaining permission from Ethics Committee of the University, this analytical - retrospective study was conducted on 62 patients with ovarian masses hospitalized in Ahwaz Imam Khomeini Hospital for surgery. The records of the patients who had been hospitalized within the years of 20112013 were analyzed and the HE4 serum level was measured by ELISA method and also the information in the patient records were recorded in demographic questionnaire. The cut-off levels of $\mathrm{He} 4$ was considered as $70 \mathrm{Pmol} / \mathrm{Lit}$. Moreover the kit He 4 EIA of Can Ag USA company was 
used. After collecting the required data using SPSS version 18 software the collected data was analyzed and for the qualitative variables the frequency and frequency percentage and for quantitative variables the mean and standard deviations were calculated. In order to evaluate hypotheses the independent t-test and ANOVA were used and the level of significance for the interpretation of the results was considered as 0.05 .

\section{Results and Discussion}

In this study, 62 patients with ovarian tumors were studied among whom 26 cases (41.9\%) were postmenopausal. Pathologically $24.2 \%$ had serous cyst adenoma, $17.7 \%$ had mucinous cyst adenoma, 3.2\% had adeno-fibroma cysts, $27.4 \%$ had serous carcinoma, $24.2 \%$ had mucinous carcinoma and $3.2 \%$ had endometrioid (Figure 1). Analyzing the patients' stage showed that $11.8 \%$ were in the first stage, $38.2 \%$ were in the second stage, $41.7 \%$ were in the third stage and $2.9 \%$ were in the fourth stage (Figure 2). $20.6 \%$ were in mild grade, $29.4 \%$ were in moderate grade and $50 \%$ were in severe grade (Figure 3 ).

In case of the type of tumor in 28 cases $(45.2 \%)$ the tumors were benign and in 34 cases (54.8) the tumors were malignant. In case of HE-4 level it was observed that the patients' average HE-4 was 276.1 \pm 316.1 and $50 \%$ of patients had HE-4 above 169 . Statistical test results showed that the HE-4 levels in postmenopausal women had no significant difference with other patients $(\mathrm{P}>$ 0.05), but the mean HE-4 level in different stages showed that by increasing the stage the HE-4 level is increased significantly $(\mathrm{P}=$ 0.007). This increase was also applied in patients' grade and HE-4 was significantly higher in severe cases the $(\mathrm{P}=0.003)$. Also HE-4 level was significantly higher in malignant patients than the benign patients $(\mathrm{P}=0.001)$ (Table 1).

Table.1 Association between HE4 and Variables

\begin{tabular}{|l|l|l|l|l|}
\hline \multirow{2}{*}{ Variable } & Sub- & \multicolumn{2}{|c|}{ HE4 level } & \multirow{2}{*}{ P-Value } \\
\cline { 3 - 4 } & Variable & Mean & SD & \\
\hline \multirow{3}{*}{ Type } & Benign & $144 / 92$ & $60 / 93$ & \multirow{2}{*}{$0 / 001$} \\
\cline { 2 - 4 } & Malignant & $384 / 04$ & $393 / 71$ & \\
\hline \multirow{3}{*}{ Stage } & Yes & $379 / 82$ & $421 / 87$ & \multirow{2}{*}{$>0 / 05$} \\
\cline { 2 - 4 } & No & $207 / 61$ & $188 / 37$ & \\
\cline { 2 - 4 } & I & $106 / 75$ & $12 / 84$ & \multirow{2}{*}{$0 / 007$} \\
\cline { 2 - 4 } & II & $187 / 8$ & $81 / 49$ & \\
\cline { 2 - 4 } Grade & III & $581 / 56$ & $472 / 78$ & \\
& IV & 884 & 0 & \\
& Mild (1) & $122 / 57$ & $58 / 92$ & \\
\cline { 2 - 4 } & Moderate (2) & $201 / 04$ & $77 / 53$ & \\
\cline { 2 - 4 } & Severe (3) & $384 / 04$ & $393 / 71$ & \\
\hline
\end{tabular}




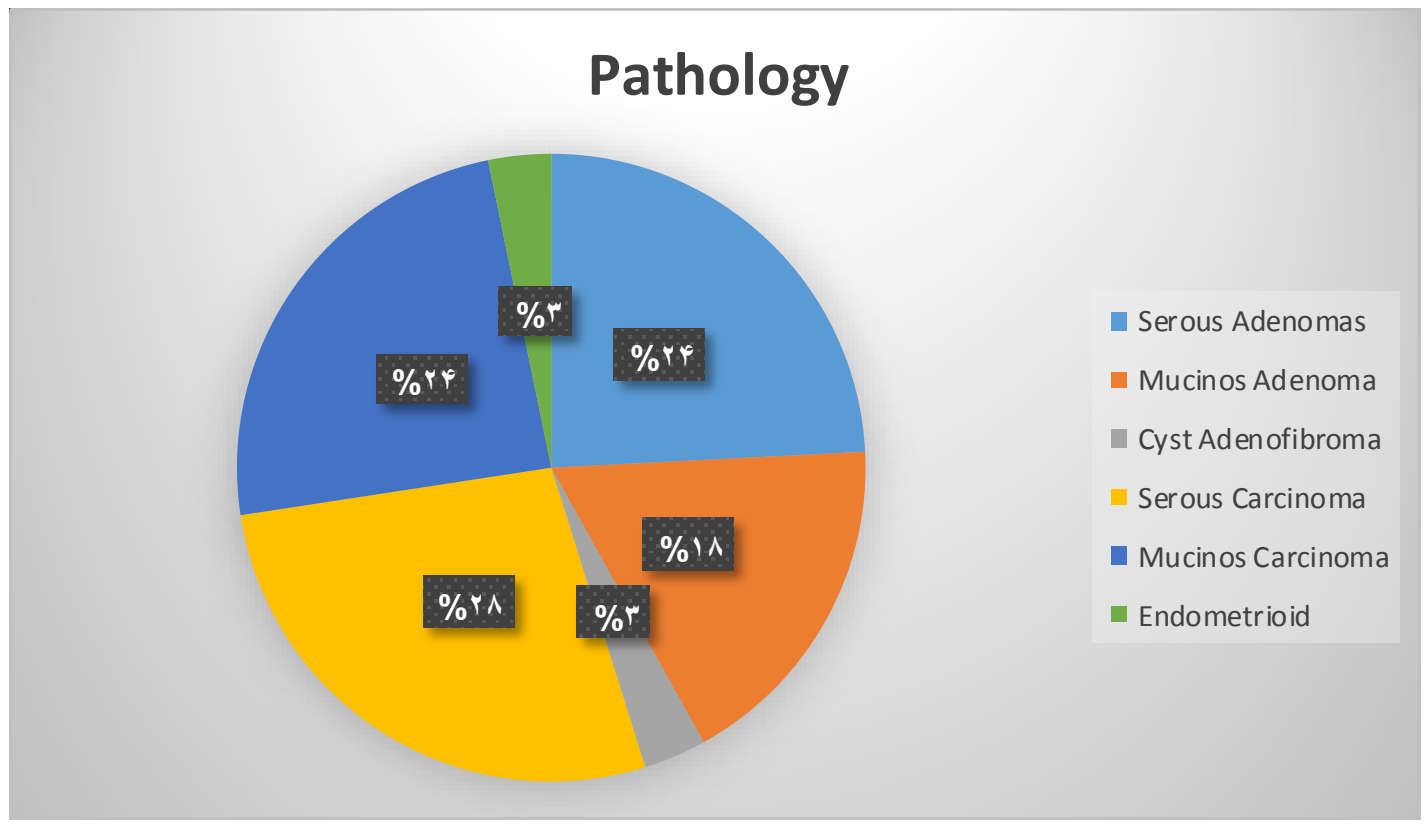

Figure.1 Frequency distribution of the pathology in patients (\%)

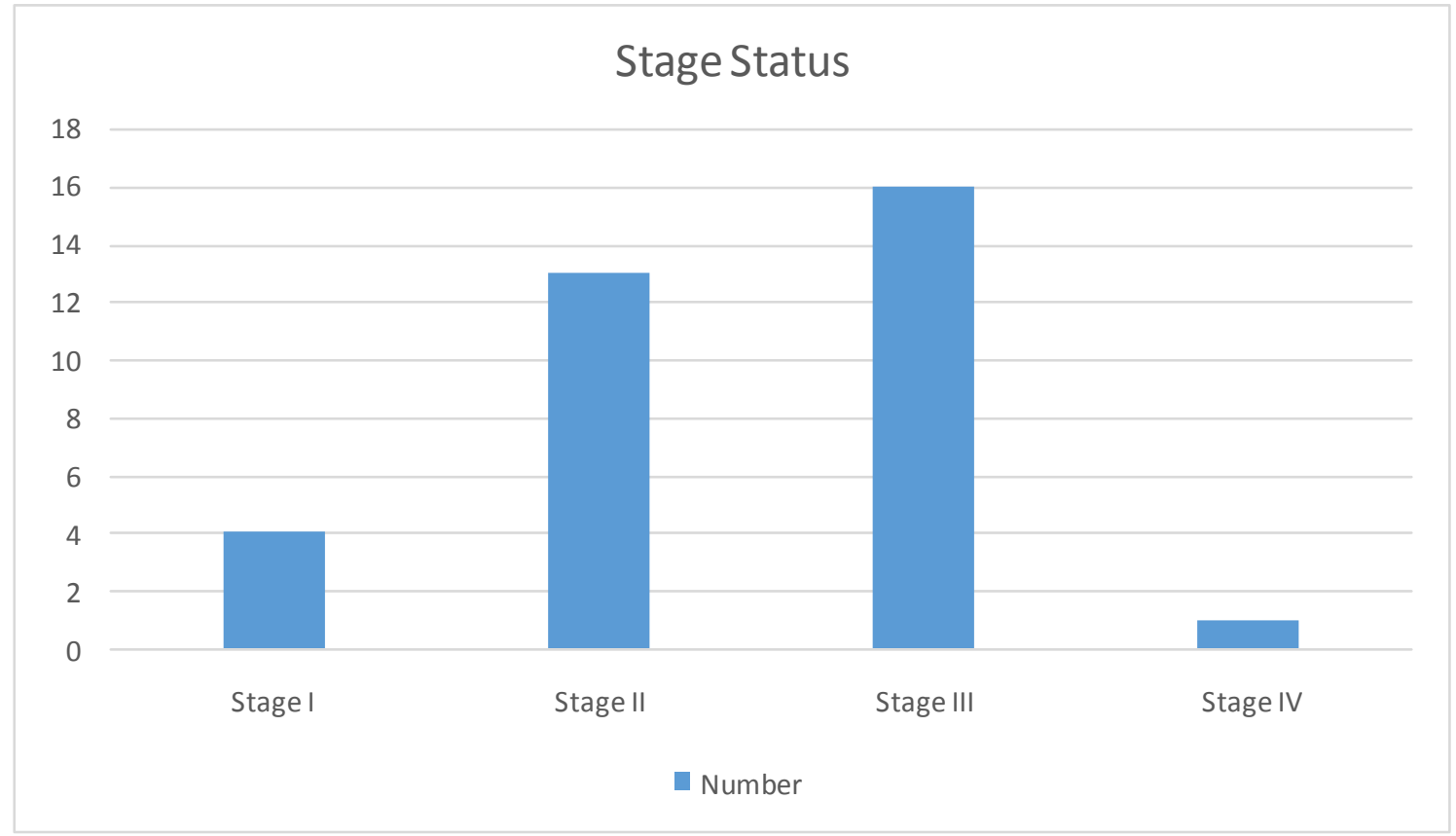

Figure.2 Frequency distribution of stage in patients with epithelial ovarian cancer (n) 


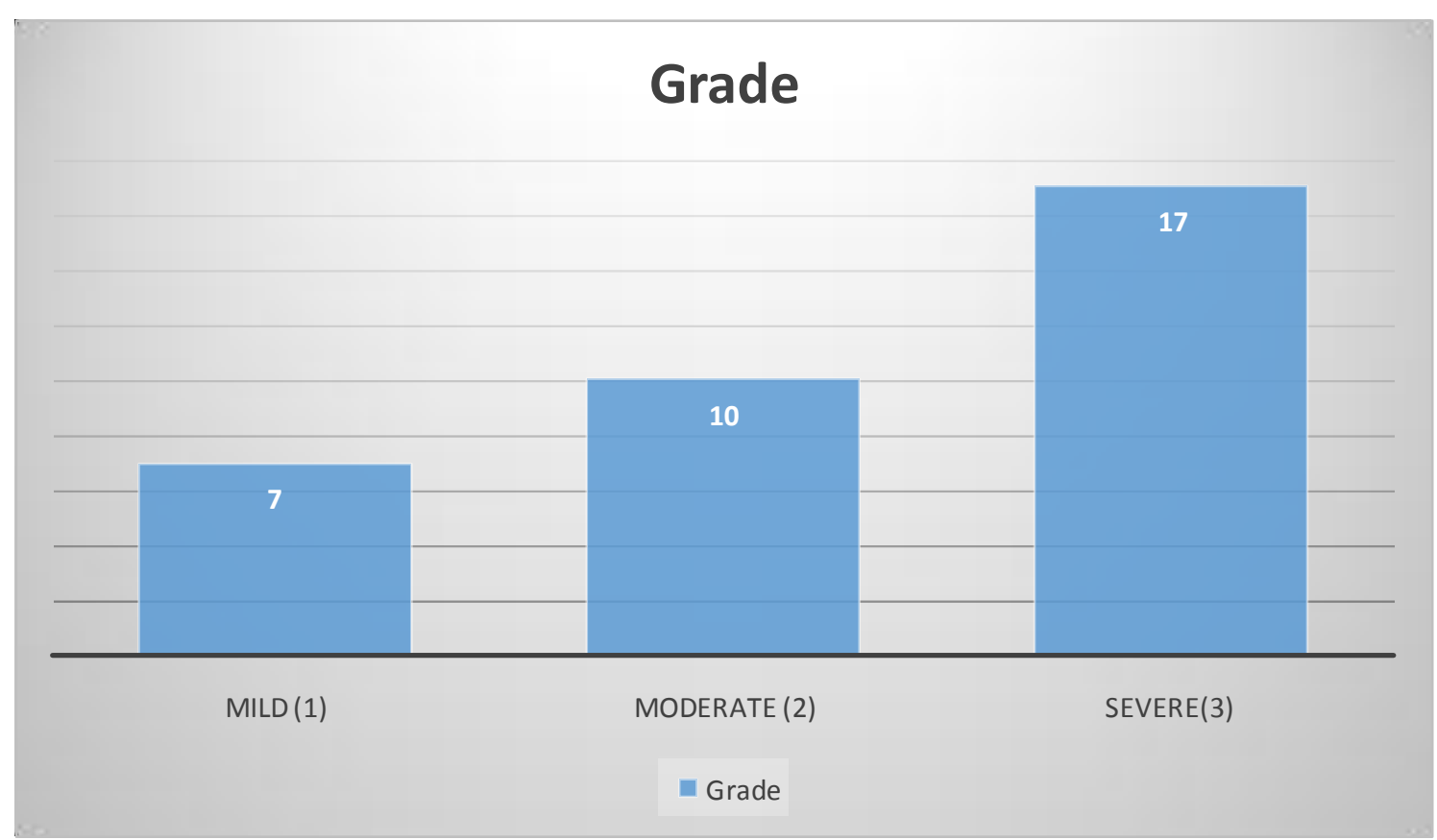

Figure.3 Frequency distribution of drade in patients with epithelial ovarian cancer

The study on the tumor markers in addition to the early diagnosis, follow up after diagnosis and treatment could predict the tumor stage based on serum levels is increasing. In line with this objective, in this study the association between preoperative serum levels of the marker of HE4 and epithelial ovarian cancer based on FIGO system to evaluate this marker in predicting the ovarian cancer stage is studied to evaluate this marker in predicting the ovarian cancer stage and help the early diagnosis of the disease in early stages and treat the patient more effectively.

In this study the patients' average HE-4 was 276.1 3 316.1. HE-4 frequency was significantly different based on benign or malignant patients $(\mathrm{P}<0.05)$ and was higher in malignant cases. Moreover HE-4 was higher in patients with greater stage and grade. Obviously to achieve an effective result of the existing data it is necessary to compare the results with the results of other similar articles. For example Throdel et al., in Canada studied the possibility of prediction of death and prognosis in patients with ovarian cancer and it was found that HE4 was an effective marker to predict mortality (Trudel et al., 2012) Of course the present study was cross-sectional and patients' mortality and factors affecting were not analyzed. Zhijun Yang et al., (2000-2013) in China performed a study to evaluate the diagnostic value of HE4serum level in ovarian cancer and concluded that HE4 has higher sensitivity (79\%) and specificity (89\%) than CA125 and therefore this study indicates that HE4 can be considered as a marker to diagnose ovarian cancer that the diagnosis between benign and malignant tumors is investigated in the present study.

Also Shin Hye Chung (2005-2010) conducted a case-control study to compare serum levels of HE4 and CA125 in ovarian cancer in Korea. Accordingly the HE4 serum levels of 32 female patients diagnosed with ovarian cancer and 34 healthy subjects 
were measured and it was concluded that the serum level of this marker was higher than the benign tumors which is consistent with the results of the present study. Also Richard et al., (2007) conducted a prospective study to use a new cancer marker in the diagnosis of ovarian cancer in 2059 patients with aviary ovarian mass and concluded that HE4 alone has the highest sensitivity in the diagnosis of ovarian cancer which is in line with the results of the present study.

In Hala Abdel-Azeez et al., (2010) for the early diagnosis of ovarian cancer in 65 patients with pelvic mass it was concluded that HE4 has the highest sensitivity in ovarian tumor diagnosis especially in the early stages of the disease (Hala et al., 2010) which confirms the results of the present study. Peek et al., (2011) conducted a cohort study to determine the HE4 serum level in patients with epithelial ovarian cancer and HE4 serum level was measured before the operation and it was finally concluded that high preoperative HE4 serum levels was associated with tumor stage and serous ovarian type of tumor. However, in the present study there was no correlation with the pathological type but there was a significant correlation with advanced stages. Hamed et al., (2011-2012) showed that HE4 serum concentration was significantly higher in malignant than the benign ovarian tumors, which is similar to the results of the present study. It is indicated in Lenhard et al., in Germany that HE4 can be used as auxiliary method in patients with ovarian cancer (Lenhard et al., 2011), which is in line with the results of the present study. Molina et al (2011) in Spain concluded that HE4 has a significant area under the curve in diagnosing the benign and malignant ovarian and uterine tumors that is consistent with the results of the present study.

In general based on the results of this study it can be concluded that HE4 is a good marker with higher efficiency to differentiate the benign and malignant ovarian tumors and more importantly in malignant patients its level is increased as the grade and stage of the disease increase and thus it is recommended to apply it in patients with a diagnosis of ovarian tumors. In the end it is proposed to conduct the future multi-center studies with higher sample volume and to confirm these results.

\section{References}

Arab, M., et al. 2010. Incidence rate of ovarian cancer in Iran in comparison with developed countries. Indian J. Cancer, 47: 322-327.

Elham, O., Hamed, et al. 2013. Significance of HE4 estimation in comparison with CA125 in diagnosis of ovarian cancer and assessment of treatment response. Diag. Pathol., 8: 11.

Firoozabadi, M.D., Rahmani, H. 2015. Prevention of nausea and vomiting: methods and utility after surgery in cancer patients? Asian Pacific J. Cancer Prevention, 16(7): 2629-35.

Hala, A., Abdel-Azeez, et al. 2010. HE4 and Mesothelin: Novel Biomarkers of Ovarian Carcinoma in Patients with Pelvic Masses. Asian Pacific J. Cancer Prevention, 11: 111-116.

Hough, C.D., et al. 2000. Large-scale serial analysis of gene expression reveals genes differentially expressed in ovarian cancer. Cancer Res., 60: 6281-6287.

IIana Cass, et al. 2008. Obstetrics and Gynecology.9 th edition. Lippincot, Williams \& Wilkins.

Khodabakhshi, R. et al. 2007. Treatment and prognosis of epithelial ovarian cancer in Iran. J. Clin. Oncol., 25(18) : 5586.

Lenhard, M., Stieber, P., Hertlein, L., et al. 2011. The diagnostic accuracy of two human epididymis protein 4 (HE4) 
testing systems in combination with CA125 in the differential diagnosis of ovarian masses. Clin. Chem. Lab. Med., 49(12): 2081-8.

Mohammad Ali Sheikhi, Ahmad Ebadi, Abdolhassan Talaeizadeh, and Hossein Rahmani. 2015. "Alternative Methods to Treat Nausea and Vomiting from Cancer Chemotherapy," Chemother. Res. Practice, vol. 2015, Article ID $818759, \quad 6$ pages, 2015. doi: $10.1155 / 2015 / 818759$

Molina, R., Escudero, J.M., Augé, J.M., et al. 2011. HE4 a novel tumour marker for ovarian cancer: comparison with CA 125 and ROMA algorithm in patients with gynaecological diseases. Tumour Biol., 32(6): 1087-95.

Moore, R.G., et al. 2008. The use of multiple novel tumor biomarkers for the detection of ovarian carcinoma in patients with a pelvic mass. Gynecol. Oncol., 108: 402-408.

Moore, R.G., et al. 2011. Utility of tumor marker HE4 to predict depth of myometrial invasion in endometrioid adenocarcinoma of the uterus. Int. J. Gynecol. Cancer, 21(7): 1185-90.

Paek, J., et al. 2011. Prognostic significance of human epididymis protein 4 in epithelial ovarian cancer. Eur. J. Obstet. Gynecol. Reprod. Biol., 158(2): 338-42.

Paek, J., Lee, S.H., Yim, G.W., Lee, M., Kim, Y.J., Nam, E.J., Kim, S.W., Kim, Y.T. 2011. Prognostic significance of human epididymis protein 4 in epithelial ovarian cancer. Eur. J. Obstet. Gynecol. Reprod. Biol., 158(2): 338-42.

Rahmani, H., Shahriary, A., Sheikhi, M.A., Ebadi, A., Davoodzadeh, H. 2016. Applications of cardio-toxicity in breast cancer: a meta-analysis. Panminerva medica.

Ren, J., et al. 2010. Tumor markers for early detection of ovarian cancer. Expert Rev. Mol. Diagn., 10(6): 787-98.

Richard, G., Moore, et al. 2008. The use of multiple novel tumor biomarkers for the detection of ovarian carcinoma in patients with a pelvic mass. Gynecol. Oncol., 108: 402-408.

Schummer, M., et al. 1999. Comparative hybridization of an array of 21,500 ovarian cDNAs for the discovery of genes overexpressed in ovarian carcinomas. Gene., 238: 375-385.

Shin Hye Chung, et al. 2013. Clinical efficacy of serum human epididymis protein 4 as a diagnostic biomarker of ovarian cancer: A pilot study. Obstet. Gynecol. Sci., 56(4): 234-241.

Trudel, D., Têtu, B., Grégoire, J., Plante, M., Renaud, M.C., Bachvarov, D., Douville, P., Bairati, I. 2012. Preoperative plasma HE4 levels could predict the occurrence of death. Gynecol. Oncol., 127(3): 511-515.

Zhijun Yang, et al. 2013. Clinical value of serum human epididymisprotein 4 assay in the diagnosis of ovarian cancer: a meta-analysis. OncoTargets and Therapy, 6: 957-966.

\section{How to cite this article:}

Fatemeh Cheraghi, Zohreh Moini and Mojgan Barati. 2016. The Relationship between Preoperative Serum Levels of HE4 and Epithelial Ovarian Cancer. Int.J.Curr.Res.Aca.Rev.4(10): 102-108. doi: http://dx.doi.org/10.20546/ijcrar.2016.410.012 